\title{
Structural evolution and kinetic study of high isotacticity poly(acrylonitrile) during isothermal pre-oxidation
}

\author{
Li Zhang, Yongqiang Dai, Yi Kai and Ri-guang Jin* \\ State Key Laboratory of Chemical Resource Engineering, Beijing University of Chemical Technology, Beijing, 100029, China
}

\author{
Article Info \\ Received 22 October 2011 \\ Accepted 31 November 2011 \\ *Corresponding Author \\ E-mail: jin.rigung@163.com
}

\section{Open Access}

DOI: http://carbonlett.org/

10.5714/CL.2011.12.4.229

This is an Open Access article distributed under the terms of the Creative Commons Attribution Non-Commercial License (http://creativecommons.org/licenses/ by-nc/3.0/) which permits unrestricted non-commercial use, distribution, and reproduction in any medium, provided the original work is properly cited.

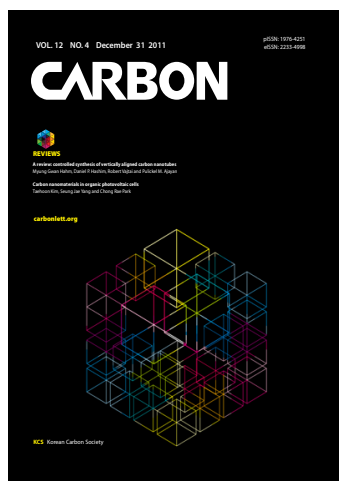

http://carbonlett.org

pISSN: $1976-4251$

elSSN: 2233-4998

Copyright $\odot$ Korean Carbon Society

\begin{abstract}
Isotactic polyacrylonitrile (PAN) with triad isotacticity of 0.53 , which was determined by ${ }^{13} \mathrm{C} \mathrm{NMR}$, using dialkylmagnesium as an initiator, was successfully synthesized. Isothermal treatment of iso-PAN was conducted in air at $200,220,250$ and $280^{\circ} \mathrm{C}$. Structural evolutions and chemical changes were studied with Fourier transformation infrared and wide-angle X-ray diffraction during stabilization. A new parameter $C N F=I_{2240 \mathrm{~cm}^{-1}} /\left(\mathrm{I}_{1595 \mathrm{~cm}}{ }^{-1}+\mathrm{f}^{*} \mathrm{I}_{1595 \mathrm{~cm}^{-1}}\right)$ was defined to evaluate residual nitrile groups. Crystallinity and crystal size were calculated with X-ray diffraction dates. The results indicated that the nitrile groups had partly converted into a ladder structure as stabilization proceeded. The rate of reaction increased with treatment temperature; crystallinity and crystal size decreased proportionally to pyrolysis temperature. The iso-conversional method coupled with the Kissinger and Flynn-Wall-Ozawa methods were used to determine kinetic parameters via differential scanning calorimetry analysis with different heating rates. The active energy of the reaction was 171.1 and $169.1 \mathrm{~kJ} / \mathrm{mol}$, calculated with the two methods respectively and implied the sensitivity of the reaction with temperature.
\end{abstract}

Key words: high isotacticity, polyacrylonitrile, stabilization, kinetics, iso-conversional

\section{Introduction}

Polyacrylonitrile (PAN)-based carbon fiber has been studied for decades [1,2]. Its excellent properties, such as high tensile strength, low weight, and low thermal expansion, make it very popular in aerospace, civil engineering, military, and motors ports, as well as in other competition sports $[3,4]$. The quality of precursor materials largely affects the final performance of carbon fibers [5], as does the thermal stabilization [6]. Earlier research on producing carbon fiber is always focused on how to improve the mechanical properties of the precursor fibers and reducing the induction period and rate of heat release during stabilization [7]. However, previous studies are rarely involved in the stereoregularity of PAN configuration, which is beneficial for cyclization during stabilization [8]. Since there are many nearby nitrile groups in high isotacticity polymer groups, this increases the cyclization degree paired with low tacticity. The high isotacticity of the precursor materials results in higher crystallinity and mechanical properties, so the final carbon fibers' properties may improve by increasing the isotacticity of precursor fibers.

Nowadays, high stereoregularity of PAN can be achieved by acrylonitrile-urea canal polymerization initiated by $\gamma$-ray irradiation [9] and matrix-anionic-polymerization $[10,11]$. In this study, high tacticity PAN, with tritacticity of 0.53 , was successfully synthesized by anionic polymerization, in non-polar solvent, using dialkylmagnesium $\left(\mathrm{R}_{2} \mathrm{Mg}\right)$ as an initiator. The high stereoregularity iso-PAN's crystallinity, used as a precursor, is effective in producing high performance carbon fiber. Moreover, the optimized pre-oxidation of isoPAN is essential, which is seldom investigated in previous studies. As pre-oxidation is a very complex process with a variety of elementary reactions such as cyclization, oxidation, 
aromatization, dehydrogenation, etc., it can hardly be studied by using a single model $[6,12,13]$. Detailed study of the chemical reactions, structural evolution and kinetics during this process will provide the necessary theoretical basis for optimizing the process and performance improvement of the final carbon fiber.

In this study, the structural evolution of high isotacticity PAN isothermal-treated at $200,220,250,280^{\circ} \mathrm{C}$ was investigated by Fourier transform infrared spectroscopy (FTIR) and wide-angle $\mathrm{X}$-ray diffraction (WXRD) respectively. Unreacted nitrile groups fractions were calculated by the peak intensity of nitrile groups at $2240 \mathrm{~cm}^{-1}$ and $1600 \mathrm{~cm}^{-1}$, which is the intensity of $\mathrm{C}=\mathrm{C}$ and $\mathrm{C}=\mathrm{N}$. Besides, thermal properties of iso-PAN were analyzed by using differential scanning calorimetry (DSC). A kinetic study was constructed from DSC dates with different heating rates of $5,10,15,20^{\circ} \mathrm{C}$. Activation energy and pre-exponential factor were determined by iso-conventional methods, viz. Kissinger and Flynn-Wall-Ozawa (FWO) methods [14].

\section{Experiment}

\subsection{Synthesis of high tacticity PAN and isothermal treatment of iso-PAN}

A $100 \mathrm{~mL}$ four-necked flask equipped with a stirrer, dropping funnel, condenser, two-way cock and thermometer was needed for polymerization. The flask was flushed with dry nitrogen for $5 \mathrm{~min}, 0.053 \mathrm{~g}(1,4$-cyclohexanediol) and $40 \mathrm{~mL}$ of Xylene were added. After dissolving the alcohol completely at $130^{\circ} \mathrm{C}$, the initiator, $\left(\mathrm{n}-\mathrm{C}_{6} \mathrm{H}_{13}\right)_{2} \mathrm{Mg}$ an $\mathrm{n}$-heptane solution, was added into the flask while stirring, causing white solvent-insoluble substances to form immediately. The solution was kept within the initiator at $138^{\circ} \mathrm{C}$ for $15 \mathrm{~min}$, then $5 \mathrm{~mL}$ of acrylonitrile (AN) were added dropwise while stirring. The polymerization started instantly and soon the solution became brown. The polymerizations were allowed for $7 \mathrm{~h}$, then hydrogen chloride $(\mathrm{HCl})$ and $\mathrm{CH}_{3} \mathrm{OH}$ solutions, as polymerization terminators, were added dropwise while stirring. After cooling to room temperature, the solution was collected by filtration through a glass filter (G3 grade), and successively washed with acetone and water, and thereafter dried in a vacuum for $24 \mathrm{~h}$, allowing white PAN powder to be obtained.

Isothermal treatments at different temperatures (200, 220, 250 and $280^{\circ} \mathrm{C}$ ) were conducted in a precision controlled tube furnace with a temperature accuracy of $1^{\circ} \mathrm{C}$. After the temperature in the furnace reached the settle temperature, the iso-PAN was placed into the furnace and pre-oxidation started immediately. Samples with different reaction degrees were obtained at various times during the reaction time at $5,10,15,30$ and $60 \mathrm{~min}$.

\section{Characterization}

\subsection{FTIR spectroscopy}

FTIR spectra of samples were recorded on a Nicolet-210 (Nicolet, USA) Spectrophotometer with a wave range of $4000-400 \mathrm{~cm}^{-1}$. Weighted samples were mixed thoroughly with a constant amount of $\mathrm{KBr}$ and palletized. As the reaction proceeded, the nitrile groups with an absorbance at $2240 \mathrm{~cm}^{-1}$, reacted to produce cyclized units containing $-\mathrm{C}=\mathrm{N}$ - and $-\mathrm{C}=\mathrm{C}$ groups, which absorbed at $1595 \mathrm{~cm}^{-1}$. Reaction degrees can be estimated via unreacted nitrile groups fractions by using the following formula:

Unreacted nitrile groups: $C N F=\frac{I_{2240 \mathrm{~cm}^{-1}}}{I_{2240 \mathrm{~cm}^{-1}}+f * I_{1590 \mathrm{~cm}^{-1}}}$

Where $\mathrm{I}_{2240 \mathrm{~cm}}{ }^{-1}$ is the absorption of nitrile groups, $\mathrm{I}_{1595 \mathrm{~cm}}{ }^{-1}$ is the absorption of $-\mathrm{C}=\mathrm{C}-$ and $-\mathrm{C}=\mathrm{N}-$ groups. $\mathrm{f}$ is the ratio of nitrile groups and cyclized groups absorptivity constants, $f=0.29$ [15].

\subsection{WAXD analysis}

The XRD pattern of high isotacticity PAN with different cyclization degrees was recorded on a Rigaku D/MAX 2500 VB2+/PC X-ray diffractometer (Japan) operated at a voltage of $40 \mathrm{kv}$ and a current of $30 \mathrm{~mA}$ with nickel-filtered $\mathrm{Cu} \mathrm{K}_{\alpha}$ radiation $(\lambda=1.54056 \AA)$, and a scanning speed of $5 \%$ min range from $5-40^{\circ}$. Crystal size (Lc) of the laterally ordered phase can be acquired from the Scherrer equation:

$$
L_{c}=\frac{K \lambda}{\beta \cos \theta}
$$

Where $\mathrm{K}$ is the apparatus constant and $\beta$ is the half-value width FWHM of the intensity versus the Bragg angle.

Crystallinity $(\mathrm{C})$ can be calculated with the following formula:

$$
L_{c}=\frac{K \lambda}{\beta \cos \theta}
$$

Where $\chi_{c}$ is the area of the crystalline portion in the XRD patterns, $\chi_{\mathrm{a}}$ is the area of the amorphous portion in the XRD patterns [7].

\subsection{DSC measurements}

DSC measurements were carried out using a STA449C calorimeter (Netzsch, Germany). Iso-PAN samples with the mass of 4-7 mg were placed into the coil which was made of an aluminum-based alloy used for DSC measurements. The same empty coil was used as a reference. The specimen was heated in the instrument with different heating rate of $5,10,15,20^{\circ} \mathrm{C} / \mathrm{min}$ from 45 to $450^{\circ} \mathrm{C}$ in an air atmosphere with a flow of $40 \mathrm{~mL} / \mathrm{min}$.

\subsection{Characteristics of high tacticity PAN}

Nuclear magnetic resonance (NMR) analysis was carried out using an Av600 wide-bore solid state NMR spectrometer equipped with $3.2 \mathrm{~mm} \mathrm{HCN}$ balun probe operating at 200.968 $\mathrm{MHz}$ for ${ }^{13} \mathrm{C}$. Spectra were collected using proton decoupling $(30 \mathrm{~ms} ; 74 \mathrm{KHz})$. During acquisition, pulse delay times were $1.745 \mathrm{~s}$, acquisition times $0.655 \mathrm{~s}$, pulse widths $5.5 \mathrm{us}\left(45^{\circ} \mathrm{pulse}\right)$. Accumulation was more than 3000 percent.

The triad tacticities $[(\mathrm{mm}),(\mathrm{mr})$ and $(\mathrm{rr})$; " $\mathrm{m}$ " and " $\mathrm{r}$ " mean "meso" and "racemo diad" sequences, respectively] were determined on the basis of the Schaefer's assignment [16,17] via ${ }^{13} \mathrm{C}$ NMR spectroscopy spectra, from the ratio of intensities of the three methane carbon peaks (26.9 ppm for mm, $27.4 \mathrm{ppm}$ 


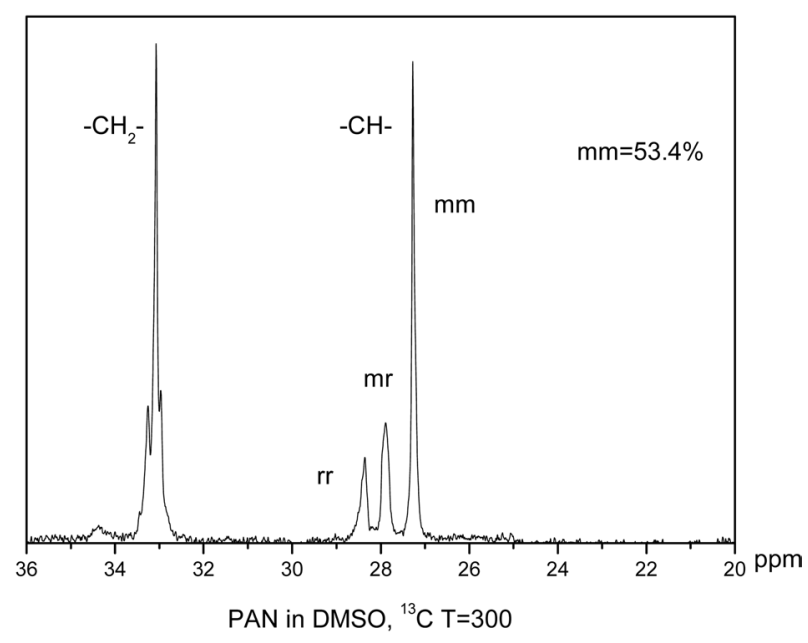

Fig. 1. ${ }^{13} \mathrm{C}$-nuclear magnetic resonance spectrum of high iso-tacticity of polyacrylonitrile (PAN).

for $\mathrm{mr}$, and $27.9 \mathrm{ppm}$ for $\mathrm{rr}$ methane carbon) in the ${ }^{13} \mathrm{C} \mathrm{NMR}$ spectrum.

By calculating the mathematical area of the three peaks, the tacticity of the polymer can be obtained: $53.4 \%(\mathrm{~mm}), 19.47 \%$ (mr), 27.13\% (rr). The higher the value of $\mathrm{mm}$ is, the more nitrile groups exist in the same plane, which greatly affects thermal stabilization of the polymer.

\section{Results and Discussion}

\subsection{Cyclization and oxidation of iso-PAN in an air atmosphere}

Pre-oxidation of iso-PAN is a complicated process, involving cyclization, oxidation and some cracking reactions. Structural evolution in the process of isothermal pre-oxidation can be examined by FTIR.

Fig. 2 shows the FTIR spectra of isothermal treated isoPAN for various times at $250^{\circ} \mathrm{C}$, among which Fig. 2a shows the spectra of the iso-PAN without heat treatment. The band at $2243 \mathrm{~cm}^{-1}$ corresponds to C-N stretching of the acrylonitrile unit in the polymer chain and the absorption bands at 2939 and $1454 \mathrm{~cm}^{-1}$ can be attributed to $\mathrm{CH}_{2}$ stretching and $\mathrm{CH}$ bending, respectively. The assignments of some other absorption bands are given as follows: $1369 \mathrm{~cm}^{-1}$ (bending of $\mathrm{CH}$ ), $1227 \mathrm{~cm}^{-1}$ (twisting vibration of $-\mathrm{CH}_{2}$ ). The weak absorption at $1632 \mathrm{~cm}^{-1}$ which is attributed to $\mathrm{C}=\mathrm{C}$ vibration, is probably due to termination by disproportionation, which can be confirmed by the discoloration of diluted bromine water [18]. The wide absorption range between $4310-3520 \mathrm{~cm}^{-1}$ corresponds to the vibration of $-\mathrm{OH}$ of unsaturated water. By comparing the spectrum of heat treated PAN for different times $(5,10,15,30$ and $60 \mathrm{~min})$, it is seen that with the extension of heat-treated time, obvious decreases happen to the $-\mathrm{CN}$ stretching band $\left(2240 \mathrm{~cm}^{-1}\right)$, which almost disappears over the long-treated time, as do the $-\mathrm{CH}_{2}$ stretching and $-\mathrm{CH}$ bending bands at 2939 and $1454 \mathrm{~cm}^{-1}$, respectively. The absorption bands appearing at $1590 \mathrm{~cm}^{-1}$ were characteristic of $\mathrm{C}=\mathrm{C}$ and $\mathrm{C}=\mathrm{N}$ combinations vibration ladder structures. As

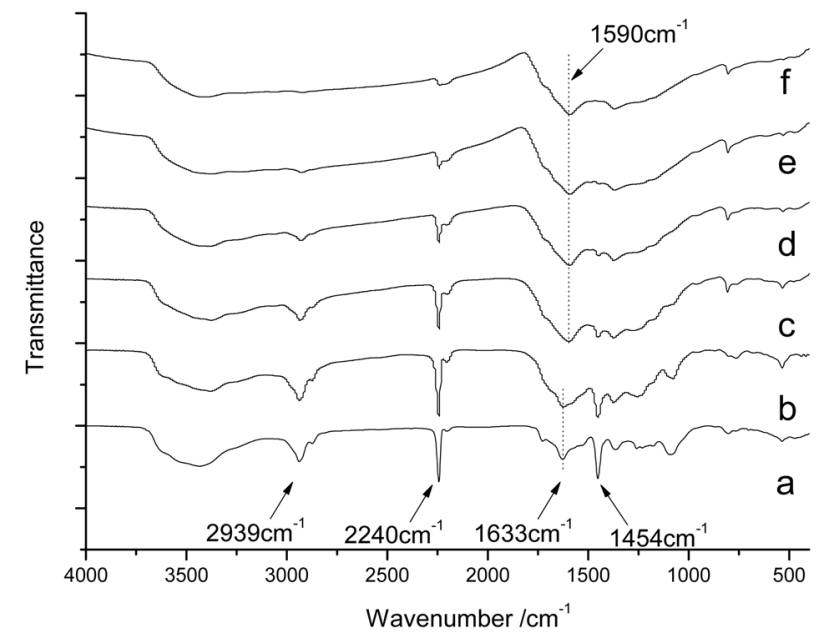

Fig. 2. Fourier transform infrared spectroscopy spectrum of isopolyacrylonitrile heated for (a) 0, (b) 5, (c) 10, (d) 15, (e) 30, (f) $60 \mathrm{~min}$ at $250^{\circ} \mathrm{C}$.

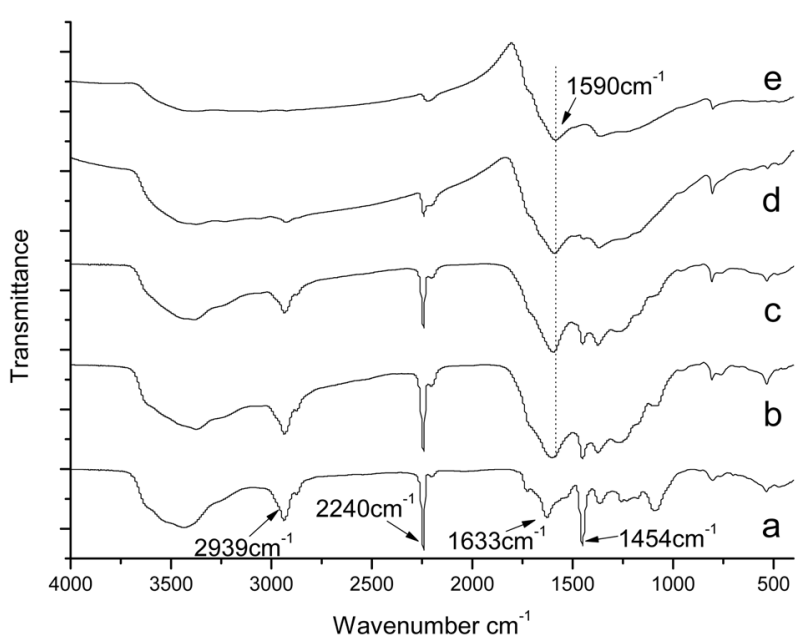

Fig. 3. Fourier transform infrared spectroscopy spectrum of isopolyacrylonitrile (PAN) heated for $30 \mathrm{~min}$ at different temperatures: (a) original iso-PAN, (b) $200^{\circ} \mathrm{C}$, (c) $220^{\circ} \mathrm{C}$, (d) $250^{\circ} \mathrm{C}$, (e) $280^{\circ} \mathrm{C}$.

shown in Fig. 2c, a wide shoulder band appears at $1730 \mathrm{~cm}^{-1}$ due to $\mathrm{C}=\mathrm{O}$ being assigned to the stretching ketone, due to oxygen uptake. The changes of spectra from (a) to (f) indicated that nitrile groups are converted into cyclized $\mathrm{C}=\mathrm{N}$ groups and cyclization, dehydrogenation, and even oxidation started progressing to a great extent as pre-oxidation proceeded.

Fig. 3 shows changes of the FTIR spectra for isothermal treated iso-PAN at different temperatures. Fig. 3a presents the original spectrum of iso-PAN before heating. There is a significant decrease in the bands of nitrile groups $\left(2240 \mathrm{~cm}^{-1}\right)$. The absorption of the nitrile groups is clear and strong in Fig. $3 \mathrm{~b}$, but indistinguishable. By Fig. 3e, it has almost disappeared, which implies that at a lower temperature, more nitrile groups remain, combined with absorption at the band of $\mathrm{C}=\mathrm{C}$ and $\mathrm{C}=\mathrm{N}$, it can be concluded that heat-treatment is a crucial factor that affects structural evolution. The less residual nitrile groups are left over, the more liner polymer chains are converted into 


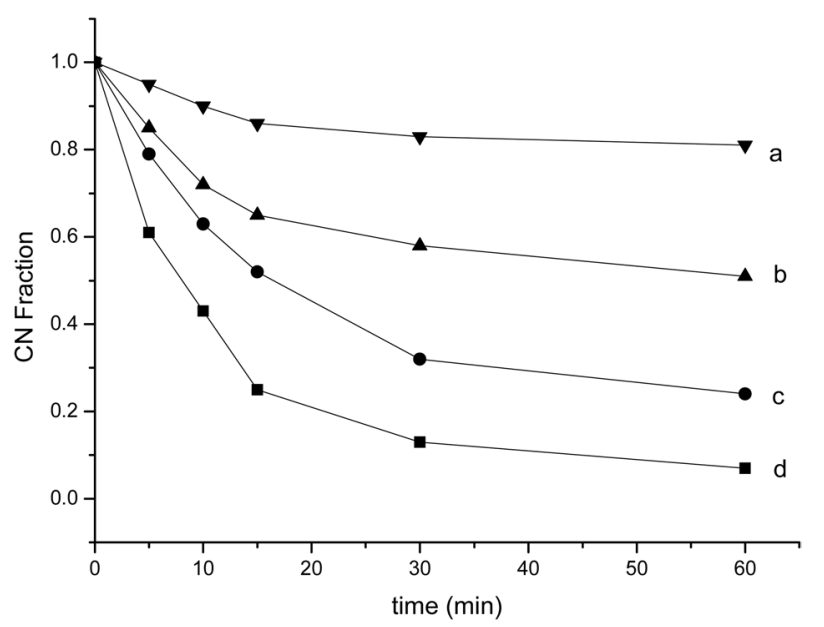

Fig. 4. The effect of temperature and time on residual nitrile groups.

cyclized structures as temperature increases. According to the formula (1), residual $\mathrm{CN}$ fractions were calculated (Fig. 4), which can measure the extent of cyclization, using the intensity at 2240 and $1590 \mathrm{~cm}^{-1}$.

Fig. 4 shows the residual nitrile groups fraction (reduced carbon nanofiber [RCNF]) for different treatment temperatures and times. All four curves followed the same trend: RCNF decreased with increasing pyrolysis temperature and time. Violent cyclization reaction exhibited an acceleratory period for the first $15 \mathrm{~min}$, where the reaction proceeded very rapidly. Most of the liner nitrile groups transformed into a cyclized structure and then entered a decay period where the reaction rate slowed down in the next $15 \mathrm{~min}$, a steric hindrance effect of the cyclized structure. When time increased, the reaction entered into a retention period, when approximately $15 \%$, $40 \%, 70 \%$ and $90 \%$ of all nitrile groups had reacted, at 200 , 220,250 and $270^{\circ} \mathrm{C}$ respectively, at a very slow rate. The persistence of unreacted nitrile groups decreased when the heat increased, which may be attributed to the formation of isolated nitrile groups which were difficult to convert into a ladder polymer structure.

\subsection{XRD analysis of isothermal treated iso-PAN}

Fig. 5 shows XRD patterns of isotactic-PAN under different heat treatment temperatures for $30 \mathrm{~min}$. A typical diffractions at $2 \theta=16.5$ and $29^{\circ}$ in Fig. 5a, (100) and (110) planes, corresponds to the hexagonal unit of the $\mathrm{C}=\mathrm{N}$ groups of the intramolecular dipole-dipole interactions among the nitrile group pairs and second-order hexagonal structure, respectively. As the stabilization goes, the intensity of the (100) peak became weaker and weaker with increasing temperature, and the (110) peaks disappeared gradually, when the temperature increased to $250^{\circ} \mathrm{C}$. There is a broader peak centered approximately at $2 \theta$ $=25^{\circ},(200)$ plane, related to the irregular sheet-like structure of the ladder polymer, swamps the secondary peak at $29^{\circ}$, as shown in Figs. 5 b-d. Stabilized at $280^{\circ} \mathrm{C}$ for $30 \mathrm{~min}$ (Fig. 5e), the diffraction of $(100)$ at $2 \theta=16.5^{\circ}$ is difficult to distinguish. This could be due to the destruction of the regularity of iso-PAN when the linear $\mathrm{C} \equiv \mathrm{N}$ in the hexagonal domain transformed into

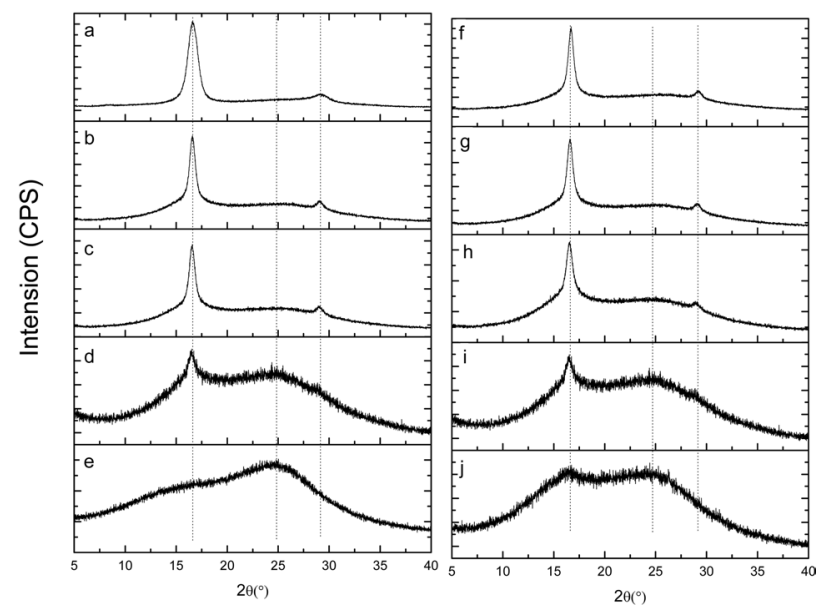

Fig. 5. Wide angle $X$-ray scattering patterns of the iso-polyacrylonitrile (PAN) heated at different temperatures: (a) original iso-PAN, (b) $200^{\circ} \mathrm{C}$, (c) $220^{\circ} \mathrm{C}$, (d) $250^{\circ} \mathrm{C}$, (e) $280^{\circ} \mathrm{C}$ for $30 \mathrm{~min}$ and various times (f) 5 , (g) 10 , (h) 15 , (i) 30, (j) $60 \mathrm{~min}$ at $250^{\circ} \mathrm{C}$.

a new ladder structure. The higher the temperature was, the higher the cyclization degree of the iso-PAN.

Figs. 5f-j shows X-ray diffraction patterns of isotactic-PAN under $250^{\circ} \mathrm{C}$ in an air atmosphere for different times. The typical diffractions at $2 \theta=16.5^{\circ}$ and $2 \theta=29^{\circ}$ corresponding to (100) and (110) planes became weaker and weaker, but a little change and minor decrease in the first $15 \mathrm{~min}$, (Figs. 5f-h), implies that a few nitrile groups of linear ordered structure were destroyed and a cyclization reaction hardly occurred in the regular domain. As shown in Fig. 5i, the intensity of (100) decreases significantly, and the intensity of (110) disappears, which indicate that the regularity of iso-PAN was destroyed and most of the linear nitrile groups were converted into ladder structures and new regular structures formed which caused the intensity at $2 \theta=25^{\circ}$ to become stronger. When prolonging the reaction time to 60 $\min$ (Fig. $5 \mathrm{j}$ ), the intensity at $2 \theta=16.5^{\circ}$ continues to decrease, and, on the contrary, the intensity at $2 \theta=25^{\circ}$ increases as the reaction proceeds, indicating more and more linear structures turned into ladder structures and new regular structures were formed.

An XRD of isothermal treated samples was measured. Of each sample, crystallinity and crystal size which were calculated via Eqs. (1-2) and the Scherren formula, as shown in Table 1. At $200^{\circ} \mathrm{C}$, the crystallinity and crystal size are at $54.38 \%$ and 121 $\mathrm{nm}$ at $5 \mathrm{~min}$., respectively. However, the crystallinity and size decreased to $42.82 \%$ and $97 \mathrm{~nm}$ at $60 \mathrm{~min}$., as the pre-oxidation proceeded during the isothermal treatment, likely, at higher treat temperatures $\left(280^{\circ} \mathrm{C}\right)$. The crystallinity and crystal size of PAN decreased from $39.59 \%$ and $96 \mathrm{~nm}$ to $8.65 \%$ and $5 \mathrm{~nm}$. These results suggest that the longer the heat treatment of PAN, the lower the crystallinity and size, and with a decrease in speed of crystallinity and size there is an increase in pre-oxidation temperature.

Crystallinity and crystal size which are closely related to the regularity of a PAN polymer chain, decreased as pre-oxidation proceeded, because linear polymer chains converted into ladder structures during isothermal heat treatment. 
Table 1. Crystalline parameters of iso-PAN during iso-thermal treatment

\begin{tabular}{|c|c|c|c|c|c|c|c|c|c|c|c|}
\hline \multicolumn{3}{|c|}{$200^{\circ} \mathrm{C}$} & \multicolumn{3}{|c|}{$220^{\circ} \mathrm{C}$} & \multicolumn{3}{|c|}{$250^{\circ} \mathrm{C}$} & \multicolumn{3}{|c|}{$280^{\circ} \mathrm{C}$} \\
\hline $\begin{array}{l}\text { Time } \\
(\min )\end{array}$ & $\begin{array}{c}\text { Crystal- } \\
\text { linity }\end{array}$ & $\begin{array}{c}\mathrm{Lc} \\
(\mathrm{nm})\end{array}$ & $\begin{array}{l}\text { Time } \\
(\mathrm{min})\end{array}$ & $\begin{array}{c}\text { Crystal- } \\
\text { linity }\end{array}$ & $\begin{array}{c}\mathrm{Lc} \\
(\mathrm{nm})\end{array}$ & $\begin{array}{l}\text { Time } \\
(\mathrm{min})\end{array}$ & $\begin{array}{c}\text { Crystal- } \\
\text { linity }\end{array}$ & $\begin{array}{c}\mathrm{Lc} \\
(\mathrm{nm})\end{array}$ & $\begin{array}{l}\text { Time } \\
(\min )\end{array}$ & $\begin{array}{c}\text { Crystal- } \\
\text { linity }\end{array}$ & $\begin{array}{c}\mathrm{Lc} \\
(\mathrm{nm})\end{array}$ \\
\hline 5 & 54.38 & 121 & 5 & 49.76 & 105 & 5 & 49.64 & 113 & 5 & 39.59 & 96 \\
\hline 10 & 52.24 & 113 & 10 & 47.91 & 94 & 10 & 40.30 & 80 & 10 & 32.53 & 24 \\
\hline 15 & 49.27 & 107 & 15 & 41.71 & 91 & 15 & 41.71 & 91 & 15 & 30.63 & 21 \\
\hline 30 & 45.84 & 108 & 30 & 40.89 & 82 & 30 & 33.70 & 22 & 30 & 10.43 & 13 \\
\hline 60 & 42.82 & 97 & 60 & 39.82 & 78 & 60 & 24.42 & 12 & 60 & 8.65 & 5 \\
\hline
\end{tabular}

Lc is the average value of apparent crystal size in all directions.

PAN: polyacrylonitrile.

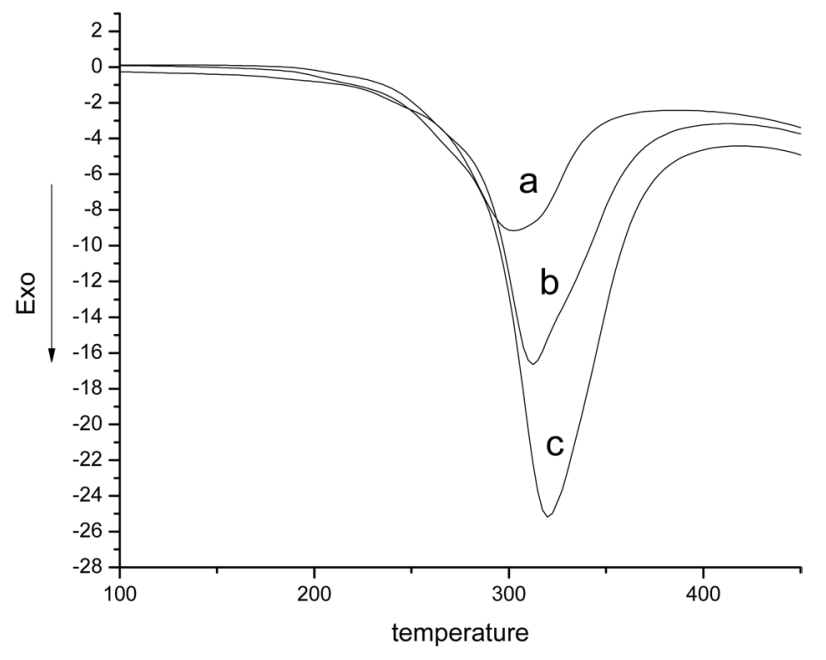

Fig. 6. Differential scanning calorimetry curves of iso-polyacrylonitrile at different heating rates: (a) 5, (b) 10, (c) $15^{\circ} \mathrm{C} / \mathrm{min}$.

\subsection{Evaluation of kinetic parameters of iso-PAN via DSC curves}

There are some methods to evaluate activation energy of preoxidation by the value of the cyclization index and aromatization through FTIR and XRD $[8,15]$ by isothermal treatment of isoPAN. However, there are some defects in each method which can only evaluate elementary reactions during pre-oxidation. However, such methods are not suitable for pre-oxidation containing elementary reactions. The iso-conversional methods involving the FWO [19] and Kissinger-Akahira-Sunose (KAS) methods [20], were used for analysis of kinetic pre-oxidation by means of DSC dates of iso-PAN at different heating rates [21].

Fig. 6 shows DSC curves of iso-PAN at different heating rates. The cyclization reaction combined with oxidation with different heating rates resulted in strong exothermic peaks in DSC analysis. The exothermic peaks wholly shifted to a higher temperature. The peak temperatures were $302.5,312$, and $319.8^{\circ} \mathrm{C}$ respectively, and the exothermic peaks became stronger and stronger. This is because the polymer has had enough time to fully react. The thermal effects and thermal inertia of the reaction increased with an increase in heating temperature.
Samples could not be heated in time, so the cyclizations only took place at higher temperatures and the exothermic peaks shifted to a higher temperature. DSC analyses were performed at different temperatures. The conversion $(\alpha)$ at any time $(t)$ was obtained from the relation:

$$
\alpha=\frac{H_{t}}{\Delta H}
$$

Where $H_{t}$ is the fractional heat of the reaction at $t \min$ and $\Delta H$ is the total enthalpy.

The rate of conversion is the linear function of the temperature-dependent rate constant, $\mathrm{k}(\mathrm{T})$, and the temperature-independent function of conversion, $\mathrm{f}(\alpha)$.

$$
\frac{d \alpha}{d t}=k(T) f(\alpha)
$$

Where $t(s)$ is time and $T(K)$ is the absolute temperature, $k(T)$ can be described by the Arrhenius equation:

$$
k=A \exp \left(-\frac{E}{R T}\right)
$$

Where $\mathrm{A}\left(\mathrm{s}^{-1}\right)$ is pre-exponential and $\mathrm{E}\left(\mathrm{Kj} \mathrm{mol}^{-1}\right)$ is the activation energy, $\mathrm{R}\left(\mathrm{Kj} \mathrm{mol}^{-1} \mathrm{~K}^{-1}\right)$ is the universal gas constant. The function $\mathrm{f}(\alpha)$ is expressed as:

$$
f(\alpha)=(1-\alpha)^{n}
$$

Where $\mathrm{n}$ is the reaction order, so the formula 3-6 can be modified to:

$$
\frac{d \alpha}{d t}=A \exp \left(-\frac{E}{R T}\right)(1-\alpha)^{n}
$$

The heating rate $\Phi$ can be described as $\phi=\frac{d T}{d t}$. Eq. (7) could be transferred to:

$$
\frac{d \alpha}{(1-\alpha)^{n}}=\frac{A}{\phi} \exp \left(-\frac{E}{R T}\right) d T
$$

The integration function of Eqs.(8) is shown as: 

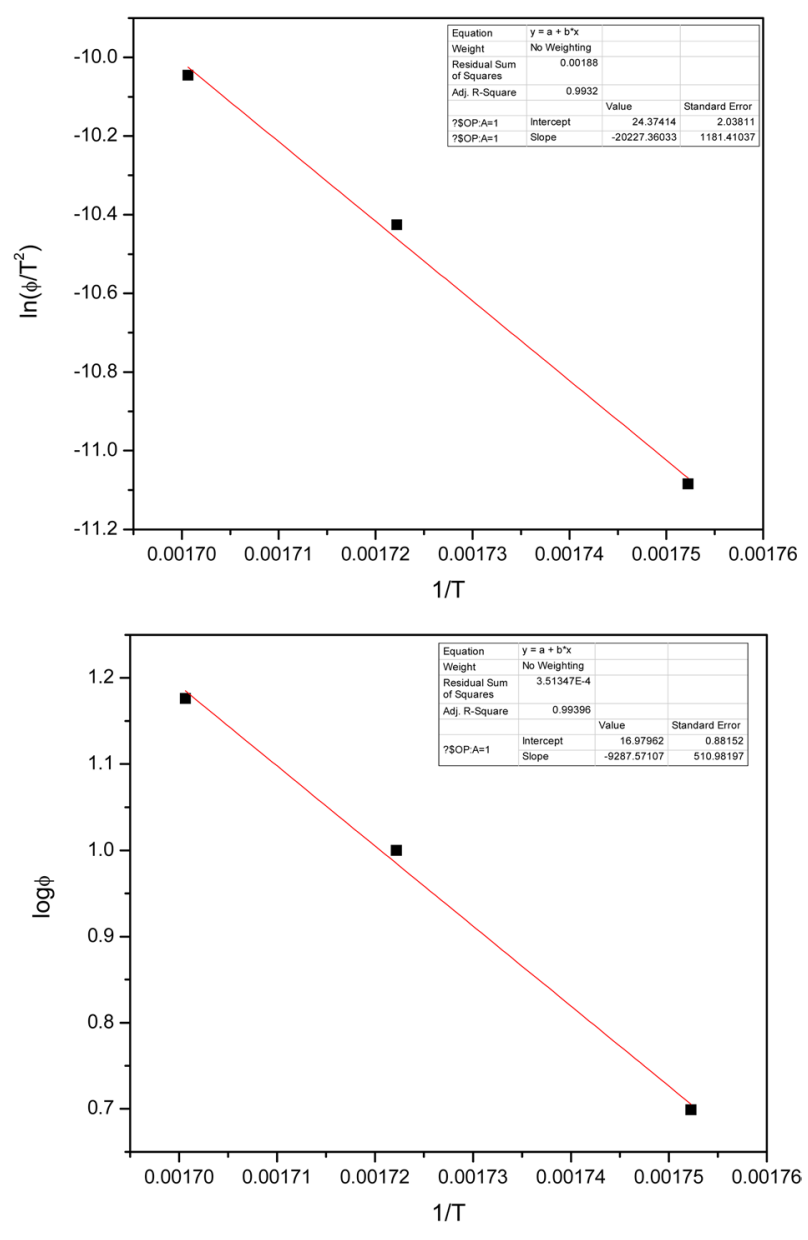

Fig. 7. Plots for determination of activation energy by (a) KissingerAkahira-Sunose method; (b) Flynn-Wall-Ozawa method corresponding to cyclization of iso-polyacrylonitrile with the heating rate of $5,10,15 \mathrm{~K} / \mathrm{min}$.

$$
g(\alpha)=\int_{0}^{\alpha} \frac{d \alpha}{(1-\alpha)^{n}}=\frac{A}{\phi} \int_{T_{0}}^{T} \exp \left(-\frac{E}{R T}\right) d T
$$

Where $\mathrm{T}_{0}$ is the starting temperature, the KAS, FWO formulas can be obtained by transforming Eq. (9) into Eqs. $(10,11)$ :

$$
\begin{aligned}
& \ln \left(\frac{\phi}{T^{2}}\right)=\ln \left[\frac{A R}{E g(\alpha)}\right]-\frac{E}{R T} \\
& \log \phi=\log \left[\frac{A E}{\operatorname{Rg}(\alpha)}\right]-2.315-0.4567 \frac{E}{R T}
\end{aligned}
$$

The advantage of the two methods is activation energy can be evaluated without knowing the reaction model and mechanism of pre-oxidation. So, the kinetic parameters can be evaluated by these methods without assuming a reaction model but rather the peak temperatures of DSC analysis at different heating rates.

Kinetic parameters of pre-oxidation by iso-conversional methods, KAS and FWO according to Eqs. (10) and (11), plots of $\ln \Phi, \ln \left(\Phi / \mathrm{T}^{2}\right)$ against $1 / \mathrm{T}$ should be straight lines, as shown in Fig. 7. Activation energies can be evaluated from the slope of the linear plots by the two methods. Pre-exponential factor A

\section{Table 2. Kinetic parameters determined by KAS and FWO methods}

\begin{tabular}{ccccc} 
& $\mathrm{E}(\mathrm{Kj} / \mathrm{mol})$ & $\mathrm{R}^{2}$ & $\bar{E}(\mathrm{Kj} / \mathrm{mol})$ & $\mathrm{A}$ \\
\hline KAS & 171.1 & 0.987 & 170.1 & $1.166 \times 10^{15}$ \\
\hline FWO & 169.1 & 0.991 & & \\
\hline
\end{tabular}

KAS: Kissinger-Akahira-Sunose, FWO: Flynn-Wall-Ozawa.

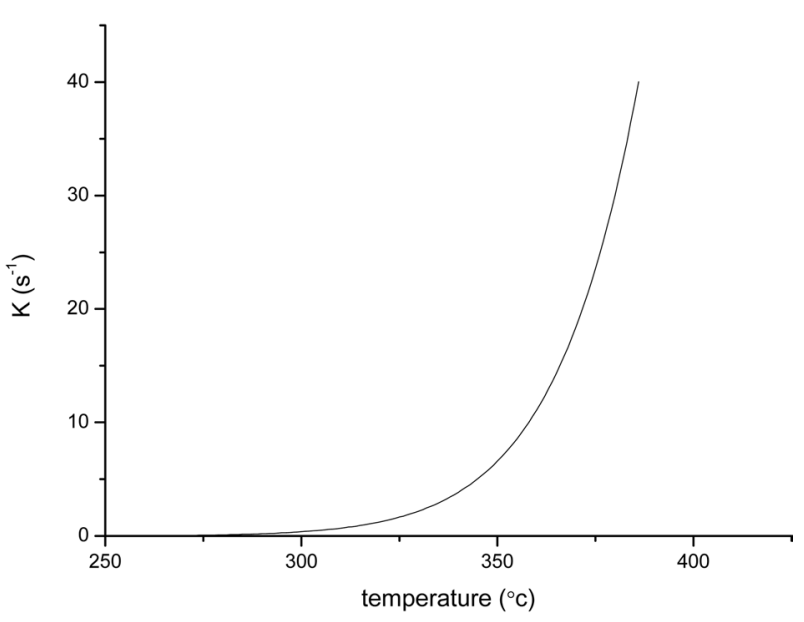

Fig. 8. Temperature dependency of rate constant (k) of the isopolyacrylonitrile.

can be calculated in both cases from the following formula [22]:

$$
A=\frac{\phi E}{R T_{P}^{2}} \exp \left(\frac{E}{R T}\right)
$$

The activation energies and pre-exponential factors of the reaction are listed in Table 2:

From the calculated data in Table 2, Ea and A values by KAS and FWO methods give nearly identical values, which indicate that the chosen iso-conversional methods are reasonable. So, the sensitivity of the pre-oxidation reaction during heat treatment can be correctly reflected by the parameters. The iso-PAN has a higher activation energy which implies that iso-PAN is more sensitive than at-PAN, via comparing the previous study to the current one [8]. The higher stereoregularity of iso-PAN makes more nitrile groups exist in the same plane, which can lower the cyclization reaction temperature and speed up the reaction rate. The rate constant $\mathrm{k}$ at different temperatures can be calculated from the Ea and A by the Arrhenius equation and is plotted in Fig. 8. It can be found that the rate of the cyclization reaction of iso-PAN increases with temperature. According the results, a centered release of heat during stabilization can be avoided by controlling the heat treatment temperature.

\section{Conclusions}

Isotacticity PAN with triad isotacticity of 0.53 , using $\mathrm{R}_{2} \mathrm{Mg}$ as an initiator was successfully synthesized. Structural evolution and chemical changes during the isothermal treatment were investigated by FTIR and WXRD analysis. The residual nitrile 
groups fractions decreased with increasing temperature, and crystallinity and crystal size decreased proportionally to the pyrolysis temperature. The kinetic parameters of the reaction were determined by iso-conversion methods, and the rate constant was calculated. The results indicate that iso-PAN is highly sensitive to treatment temperature. This study gives scientific support for controlling stabilization temperatures which is beneficial for producing high performance carbon fibers.

\section{References}

[1] Shindo A. Studies on Graphite Fiber Report of the Government Industrial Research Institutes Osaka, Agency of Industrial Science and Technology, Osaka, Japan, 317 (1961).

[2] Shindo A. 130. On the carbonization of polyacrylonitrile fiber. Carbon, 1, 391 (1964). http://dx.doi.org/10.1016/0008-6223(64)90421-x.

[3] Edie DD. The effect of processing on the structure and properties of carbon fibers. Carbon, 36, 345 (1998). http://dx.doi.org/10.1016/ s0008-6223(97)00185-1.

[4] Ogawa H. Architectural application of carbon fibers development of new carbon fiber reinforced glulam. Carbon, 38, 211 (2000). http://dx.doi.org/10.1016/s0008-6223(99)00146-3.

[5] Bahl OP, Mathur RB, Kundra KD. Structure of PAN fibres and its relationship to resulting carbon fibre properties. Fibre Sci Technol, 15, 147 (1981). http://dx.doi.org/10.1016/0015-0568(81)90067-1.

[6] Rahaman MSA, Ismail AF, Mustafa A. A review of heat treatment on polyacrylonitrile fiber. Polym Degrad Stab, 92, 1421 (2007). http://dx.doi.org/10.1016/j.polymdegradstab.2007.03.023.

[7] Devasia R, Reghunadhan Nair CP, Sadhana R, Babu NS, Ninan $\mathrm{KN}$. Fourier transform infrared and wide-angle X-ray diffraction studies of the thermal cyclization reactions of high-molar-mass polyacrylonitrile-co-itaconic acid. J Appl Polym Sci, 100, 3055 (2006). http://dx.doi.org/10.1002/app.23705.

[8] Xu ZX, Xu J, Xu LH, Dai YQ, Xue LW, Jin RG. Kinetic study of cyclization of high-tacticity polyacrylonitrile heat-treated under air atmosphere via XRD. Polymer (Korea), 32, 150 (2008).

[9] Yamazaki H, Miyazaki Y, Kamide K. Stereospecific polymerization of acrylonitrile using acrylonitrile-urea canal complex initiated by $\gamma$-ray irradiation. Roles of radical chain transfer reagents. Polym J, 23, 765 (1991). http://dx.doi.org/10.1295/polymj.23.765.
[10] Wan AJ, Zhao CX, Qian BJ. Optimum seeking method for synthesizing high stereoregular polyacrylonitrile. Polym Mater Sci Eng, 17, 48 (2001).

[11] Wu C, Wan A, Zhao J, Synth Technol Appl, 15, 1 (2000).

[12] Bashir Z. A critical review of the stabilisation of polyacrylonitrile. Carbon, 29, 1081 (1991). http://dx.doi.org/10.1016/0008-6223(91) 90024-D.

[13] Dalton S, Heatley F, Budd PM. Thermal stabilization of polyacrylonitrile fibres. Polymer, 40, 5531 (1999). http://dx.doi. org/10.1016/s0032-3861(98)00778-2.

[14] Chen C, Ma X, Liu K. Thermogravimetric analysis of microalgae combustion under different oxygen supply concentrations. Appl Energy, 88, 3189 (2011). http://dx.doi.org/10.1016/j.apenergy. 2011.03.003.

[15] Collins GL, Thomas NW, Williams GE. Kinetic relationships between heat generation and nitrile consumption in the reaction of poly(acrylonitrile) in air at $265^{\circ} \mathrm{C}$. Carbon, 26, 671 (1988). http:// dx.doi.org/10.1016/0008-6223(88)90070-X.

[16] Kamide K, Ono H, Hisatani K. Stereospecifity in the polymerization of acrylonitrile using anionic initiators including dialkylmagnesium. Polym J, 24, 917 (1992). http://dx.doi.org/10.1295/polymj.24.917.

[17] Ono H, Hisatani K, Kamide K. NMR spectroscopic study of side reactions in anionic polymerization of acrylonitrile. Polym J, 25, 245 (1993). http://dx.doi.org/10.1295/polymj.25.245.

[18] Bajaj P, Sreekumar TV, Sen K. Effect of reaction medium on radical copolymerization of acrylonitrile with vinyl acids. J Appl Polym Sci, 79, 1640 (2001). http://dx.doi.org/10.1002/1097-4628 (20010228)79:9<1640::aid-app140>3.0.co;2-7.

[19] Ozawa T. A new method of analyzing thermogravimetric data. Bull Chem Soc Jpn, 38, 1881 (1965). http://dx.doi.org/10.1246/ bcsj.38.1881.

[20] Kissinger HE. Reaction kinetics in differential thermal analysis. Anal Chem, 29, 1702 (1957). http://dx.doi.org/10.1021/ac60131a045.

[21] Tiptipakorn S, Damrongsakkul S, Ando S, Hemvichian K, Rimdusit $\mathrm{S}$. Thermal degradation behaviors of polybenzoxazine and siliconcontaining polyimide blends. Polym Degrad Stab, 92, 1265 (2007). http://dx.doi.org/10.1016/j.polymdegradstab.2007.03.021.

[22] Reghunadhan Nair CP, Krishnan K, Ninan KN. Differential scanning calorimetric study on the Claisen rearrangement and thermal polymerisation of diallyl ether of bisphenols. Thermochim Acta, 359, 61 (2000). http://dx.doi.org/10.1016/s0040-6031(00) 00504-9. 